International Journal of Engineering \& Technology, $7(2.13)(2018)$ 145-149
SPC

\title{
Development of the mathematical model for the process of oil raw materials pressing
}

\author{
Ainura Kairbayeva ${ }^{1 *}, V_{i t a l i i}$ Vasilenko $^{2}$, Seit Dzhinguilbayev ${ }^{1}$, Lyazzat Baibolova $^{1}$, Larisa Frolova ${ }^{2}$ \\ ${ }^{1}$ Almaty Technological University, Almaty, Kazakhstan \\ ${ }^{2}$ Voronezh State University of Engineering Technologies, Voronezh, Russia \\ *Corresponding author E-mail: ainurmapp@mail.ru
}

\begin{abstract}
The mathematical model for the process of oily raw materials pressing with single-screw oil press has been developed, which makes it possible to calculate the main design parameters of an oil press for extracting oil from oil crop seeds.

This model of pressing describes the process of moving meal in the pressure zone and expression of oil in the pressing cage of the screw press, taking into account the following assumptions: porous skeleton and oil have the same density, they have no chemical interaction, the process of oil filtration occurs under isothermal conditions with laminar conditions of motion.

The developed mathematical model corresponds to physical meaning of the occurring phenomena and can be used to analyze the work of active presses, and at the design stage.
\end{abstract}

Keywords: Pressing; Oil Crops; Mathematical Model; Heat and Mass Transfer.

\section{Introduction}

The expression process for many years have been the main process of the press method for the production of vegetable oils. Today despite the widespread implementation of the extraction method, it plays an important role in the technical development of fat and oil industry.

The current works on the expression process can be briefly summarized as follows: A lot of attention was paid to the problems connected with the pressure developing during expression. Thus, theoretical equations for the distribution of pressures in screw presses were proposed by N.D. Alekseev, V.A. Maslikov and D.M. Rub. Experimental data on the distribution of pressures was obtained by A.A. Medvedev and G.V. Zarembo and M.R. Shamsutdinov. The empirical equations connecting the pressure to the output of the oil (oilseed cake) were proposed. The work of Koo E.S. enabled to identify an empirical equation that connects oil output with pressure, duration of the expression, oil viscosity, and its content in the raw material. In the works of V.I. Sokolov, V.V. Dormenko and N.I. Isaeva the expression process is considered as filtration in a deformable porous medium [1 3].

The literature data indicates that despite a significant amount of research, there is no complete understanding of oilseeds pressing mechanisms, and there is no mathematical model that describes the process with all parameters taken into account. This situation slows down the use of the improved methods, and also restrains the use of new promising methods and development of the effective equipment that would intensify all stages of a rational, resource saving technological process [4], [5].

The development of suitable technologies for the processing of agricultural products requires complete knowledge of biomaterial technical properties. We have the following engineering properties of melon seeds of three species: C. edulis, C. vulgaris and C. lanatus were researched [6]
The degree of seeds humidity during shattering affects the effectiveness of shuttering, this process and its mathematical model have been studied [7].

A comparative analysis of the effect on the output of oil from the melon seeds, the parameters of the seeds at leaching have been studied [8].

The kinetics of water absorption by melon seeds have been studied [9]. The data presented in this article will be useful for the design of the sorption process and equipment.

\section{Object and methods of research}

To study the mechanism of heat and mass transfer during the pressing of oilcrop seeds (flax, rapeseed, camelina, safflower), a mathematical model of the process was developed (Fig. 1).

Transferring of oil raw materials occurs in pressure zone I of the screw production with the pressure required for the oil expression process. Zone II is a filtration zone, where, under the effect of the created pressure, oil is separated from meal with the use of pressing cage lattice.

The processed material can be represented as a two-component system of fiber in the form of a porous skeleton and oil that fills its freespace.

If we assume that the working space in the second zone is completely filled with raw materials, then as the screw rises pressure, the skeleton volume decreases, and the volume of oil in the space outside the skeleton increases by the same amount, which allows us to consider the entire two-component system as incompressible medium. It is obvious that pressure at the outlet from the injection zone will determine the amount of "free" oil in raw material, which will be taken as one of initial parameters in mathematical description of the filtering process (Fig. 2). 
Due to the complexity of the phenomena occurring during the raw materials processing in pressing cage zone of the screw presses, analytical approaches for mathematical modeling of oil expression in a general setting are not applicable [10,4]. In connection with this, we make assumptions that greatly simplify formulation of the problem and allow us to derive the calculated dependencies corresponding to physical essence of the occurring processes.

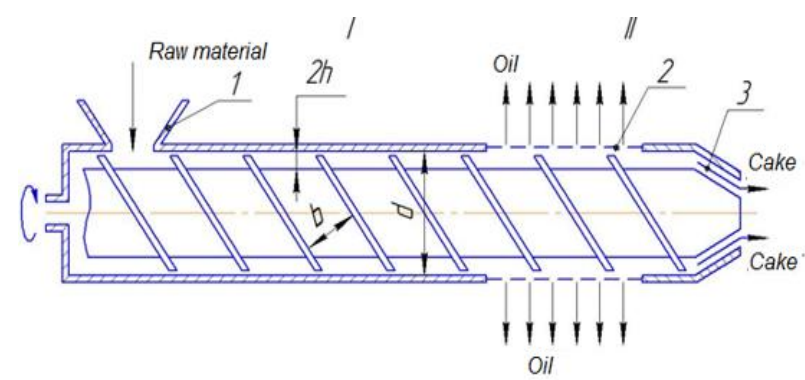

Fig. 1: Principle Diagram of the Screw Press: One - Raw Materials Receiver; two - Screw Shaft; Three - Pressing Cage Lattice; four - Outlet.

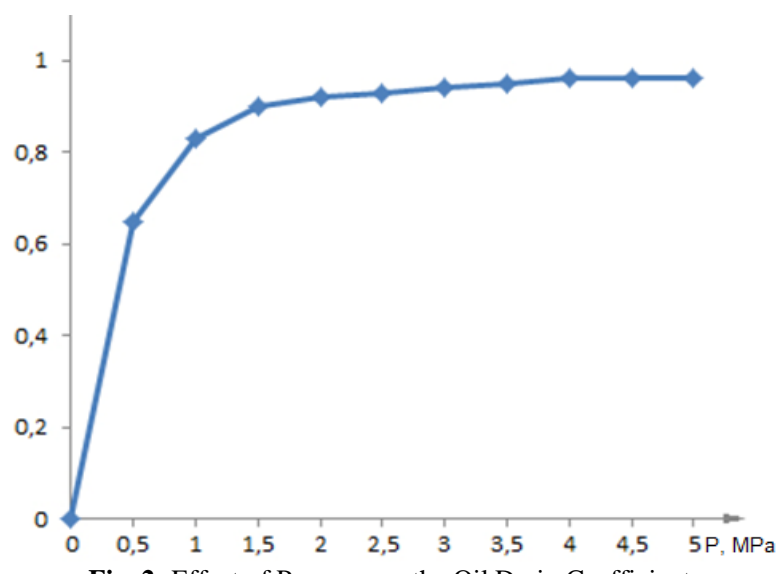

Fig. 2: Effect of Pressure on the Oil Drain Coefficient

Following [10,11], we assume that the porous skeleton and oil have same density, they do not have chemical interaction, and the process of oil filtration occurs under isothermal conditions.

Since viscosity of the filtering medium is sufficiently large, and dimensions of the pores through which mobile phase occurs is small and the filtration process is considered to occur under laminar motion. In this regard, we use Darcy's law to describe the process of oil expression, considering that inertia effects and viscous forces can be neglected, as well as an axial component of filtering speed. In the absence of gravity action for an incompressible fluid, the Darcy's law says [1]:

$$
\mathrm{V}_{\mathrm{F}}(\mathrm{z})=-\frac{\mathrm{k}}{\mu} \cdot \frac{\mathrm{dP}(\mathrm{z})}{\mathrm{dy}}
$$

where $v_{f}$ is the velocity of the fluid in a porous medium;

$\mu$ - dynamic viscosity;

$\mathrm{dP}(\mathrm{z}) /$ dy is radial pressure gradient;

$\mathrm{K}$ - Permeability, which integrally takes into account channels structure of the processed meal in the entire working volume of the pressing cage.

Let us unfold a screw channel on the plane in the expression zone and consider an elementary parallelepiped with thickness dz (fig.3). If the reference point is located on the screw the shaft as shown in Fig. 3, the upper wall of the channel is fluid-permeable. Due to the fact that the drain of the cake through the pressing cage lattice occurs into atmosphere, radial pressure gradient is determined by the excess pressure $\mathrm{P}(\mathrm{z})$ near fixed channel wall and assuming its consistency along the length of the filtered layer is defined as

$-\frac{\mathrm{dp}(\mathrm{z})}{d y}=\mathrm{P}(\mathrm{z}) / \mathrm{h}$

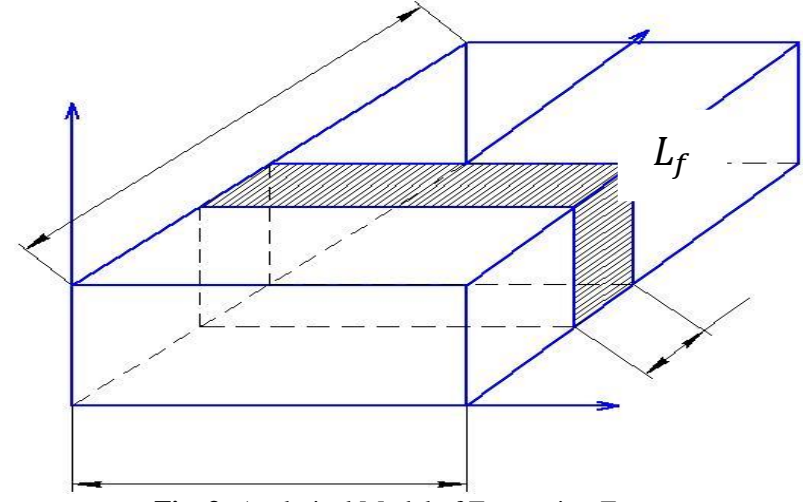

Fig. 3: Analytical Model of Expression Zone.

Since pressure along the screw shaft in the pressing cage increases, we adopt for the description the following exponential dependence

$P(z)=P_{f} \cdot e^{a z}$

where $\mathrm{P}_{\mathrm{f}}$ is pressure on the inlet to the filtration zone, and coefficient at the exponent is determined through the pressure $\mathrm{P}_{\mathrm{k}}$ at the end of the pressing cage with a length $\mathrm{L}_{\mathrm{f}}$

$\mathrm{a}=\ln \left(\frac{\mathrm{P}_{\mathrm{k}}}{\mathrm{P}_{\mathrm{f}}}\right) / \mathrm{L}_{\mathrm{f}}$

The flow of oil entering the elementary parallelepiped through its left side during the stationary mode of press operation is equal to

$\mathrm{m}(\mathrm{z})=\mathrm{V}_{\mathrm{zcp}} \cdot \mathrm{hb} \cdot \mathrm{c}(\mathrm{z})$

where $\mathbf{V}_{\mathbf{z a v e}}$-an average speed of oil along the channel section; $\mathrm{h}$ and $\mathrm{b}$ - height and width of the channel;

$\mathbf{c}(\mathbf{z})$ - local volume concentration of oil in the peeling.

Flows of the target component directed outward from the considered elementary volume are equal to:

with filtrate

$\mathrm{m}_{\mathrm{f}}(\mathrm{z})=\mathrm{V}_{\mathrm{F}}(\mathrm{z}) \cdot \mathrm{c}(\mathrm{z}) \cdot \mathrm{b} \cdot \mathrm{dz}$

With mea

$\mathrm{m}(\mathrm{z}+\mathrm{dz})=\mathrm{V}_{\mathrm{zave}} \cdot \mathrm{hb} \cdot \mathrm{c}(\mathrm{z}+\mathrm{dz})$

The flow balance equation for the elementary parallelepiped under consideration will be:

$\mathrm{m}(\mathrm{z})=\mathrm{m}_{\mathrm{f}}(\mathrm{z})+\mathrm{m}(\mathrm{z}+\mathrm{dz})$

If $\mathrm{c}(\mathrm{z})$ is a continuous function, then

$c(z+d z)=c(z)+\frac{d c(z)}{d z} d$

then taking into account equations (1) and (3), the relation (9) takes the following form:

$\mathrm{V}_{\text {zave }} \mathrm{h} \frac{\mathrm{dc}(\mathrm{z})}{\mathrm{d}(\mathrm{z})}=-\frac{\mathrm{k}}{\mu \mathrm{h}} \mathrm{e}^{\mathrm{az}} \cdot \mathrm{c}(\mathrm{z})$

Separating variables in 10 , we obtain a mathematical model of filtering process in the pressing cage:

$\frac{\mathrm{dc}(\mathrm{z})}{\mathrm{c}(\mathrm{z})}=-\frac{\mathrm{kP}_{\mathrm{F}}}{\mu \mathrm{h}^{2} \mathrm{~V}_{\mathrm{zave}}} \cdot \mathrm{e}^{\mathrm{az}} \mathrm{dz}$

$\mathrm{C}(0)=\mathrm{C}_{0}$

Integration of equation (11) with account of the single-valued condition (12), gives the following dependency for the distribution of "free" oil along the pressing cage: 


$$
\tilde{N}(z)=C_{0} \cdot \exp \left[\frac{K \cdot P_{f}}{\mu \cdot h^{2} \cdot V_{z c p} \cdot a} \cdot\left(1-e^{a z}\right)\right]
$$

Obviously, the final concentration of the target product in the meal at the outlet from the filtration zone is:

$$
\tilde{N}\left(L_{f}\right)=C_{\mathrm{o}} \cdot \exp \left[\frac{K \cdot\left(P_{f}-P_{K}\right)}{\mu \cdot h^{2} \cdot V_{z c p} \cdot a}\right]
$$

With the help of equation (13) it is possible to find concentration of the filtered oil at any point of the pressing cage by the formula:

$$
\tilde{N}_{\hat{o}}(z)=C_{0} \cdot\left(1-\frac{e^{-\frac{k \cdot P_{f}}{\mu \cdot h^{2} \cdot V_{z c p} \cdot a} \cdot e^{a z}}}{e^{-\frac{k \cdot P_{f}}{\mu \cdot h^{2} V_{z c p} \cdot a}}}\right)
$$

An average integral value of the filtered oil concentration along the length of the filtration zone:

$$
\overline{\tilde{N}}_{\hat{o}}=\frac{1}{L_{f}} \int_{0}^{L_{f}} C_{\hat{o}}(z) \cdot d \cdot z
$$

It is necessary to calculate productivity of the screw press with oil. Using equation (15), an average value of the filtered oil concentration according to (16) is:

$$
\overline{\tilde{N}}_{\hat{o}}=\tilde{N}_{0}\left\{1-\frac{e^{\frac{K \cdot P_{f}}{\mu \cdot h^{2} V_{z c p} \cdot a}}}{L_{f} \cdot a} \cdot\left[\tilde{A}\left(0, \frac{K \cdot P_{f}}{\mu \cdot h^{2} \cdot V_{z c p} \cdot a}\right)-\tilde{A}\left(0, \frac{\hat{E} \cdot P_{K}}{\mu \cdot h^{2} \cdot V_{z c p} \cdot a}\right)\right]\right\}
$$

Where ${ }^{G}(a, z)$ is incomplete gamma-function?

According to the flow rate equation, a press capacity for the filtered oil will be:

$$
c_{m}=v_{f}^{-} \cdot S_{f} \cdot \bar{c}_{f}
$$

$\boldsymbol{G}_{i}=\bar{V}_{\hat{o}} \cdot S_{\hat{o}} \cdot \overline{\boldsymbol{C}}_{\hat{o}}, \bar{V}_{\hat{o}} \ldots$ an average value of the filtering speed along the entire length of expression zone, which was determined by its integral average value.

$$
\bar{V}_{\hat{o}}=\frac{\boldsymbol{l}}{\boldsymbol{L}_{f}} \int_{\mathrm{o}}^{L_{f}} \boldsymbol{V}_{\hat{o}}(z) \cdot \boldsymbol{d} \cdot z
$$

Substituting equation (1) into (14) after integration, we obtain:

$$
\bar{V}_{\hat{o}}=\frac{K \cdot\left(P_{K}-P_{f}\right)}{L_{f} \cdot \mu \cdot h \cdot a}
$$

Using relations (11) and (14) in (12), we obtain the working formula for calculating productivity of a screw press with oil:

$$
\tilde{N}_{i}=\frac{K \cdot\left(P_{K}-P_{f}\right)}{\mu \cdot h \cdot a} \cdot b \cdot \tilde{N}_{0}\left\{1-\frac{e^{\frac{K \cdot P_{f}}{\mu \cdot P_{z p} \cdot a}}}{L_{f} \cdot a} \cdot\left[\tilde{A}\left(0, \frac{K \cdot P_{f}}{\mu \cdot h^{2} \cdot V_{z c p} \cdot a}\right)-\tilde{A}\left(0, \frac{\hat{E} \cdot P_{K}}{\mu \cdot h^{2} \cdot V_{z c p} \cdot a}\right)\right]\right\}
$$

If productivity of the press is set to oil, then on the basis of relation (21) it is possible to verify the value of permeability of oil raw materials, and then other parameters of the filtration process.

The following initial data were used for the calculations:

- $\quad$ Diameter of the pressing cage - $d=0,07 \mathrm{~m}$;

- $\quad$ Screw pitch - $\boldsymbol{H}=\mathbf{O}, \mathbf{0 2 5} \mathrm{m}$;

- Screw channel height- $\boldsymbol{h}=\mathbf{0}, 008 \mathrm{~m}$;

- Screw channel width- $\boldsymbol{b}=\mathrm{O}, \mathrm{O} 248 \mathrm{~m}$;
- Length of the expression zone $-L_{2}=0,1 \mathrm{~m}$.

Let us determine the length of a screw channel in the filtering zone by formula

$$
L_{f}=m \cdot L_{b}
$$

It is necessary to calculate the number $m$ in pressing cage and the length of the flight screw $\boldsymbol{L}_{b}$.

Since

$$
\begin{aligned}
& m=\frac{L_{2}}{\boldsymbol{H}}=\frac{\mathbf{O}, \mathbf{1}}{\mathbf{O}, \mathbf{O} 25}=\mathbf{4} \\
& L_{b}=\sqrt{H^{2}+\left(\pi \cdot d^{2}\right)}=\sqrt{0,025^{2}+\left(3,14 \cdot 0,67^{2}\right)}=0,221 \mathrm{M},
\end{aligned}
$$

Then the magnitude $L_{f}$ according to (22) is equal to

$$
L_{f}=4 \cdot 0,221=0,884
$$

Then

$\mathrm{S}_{\mathrm{f}}=0,0248 \cdot 0,884=0,0219 \mathrm{~m}^{2}$

If the screw press has a mass productivity of $\mathrm{G}=6,67 \cdot 10-3 \mathrm{~kg} / \mathrm{s}$ according to raw materials, then assuming feed density equal to $\mathrm{p}=$ $867 \mathrm{~kg} / \mathrm{m} 3$, its corresponding volumetric productivity will be

$\mathrm{V}=\mathrm{G} / \mathrm{p}=6,67 \cdot 10^{-3} / 867=7,69 \cdot 10^{-6} \mathrm{~m}^{3} / \mathrm{s}$.

Average speed of meal in the section of screw channel will be equal to

$\mathrm{V}_{\text {zave }}=\mathrm{V} / \mathrm{b} \cdot \mathrm{h}=7,69 \cdot 10^{-6} / 0,0248 \cdot 0,008=0,0388 \mathrm{~m} / \mathrm{s}$.

According to the pressure at the inlet to the filtration zone $p_{z}=0,5$ $\mathrm{MPa}$ from Fig. 2, we determine oil drain coefficient $\beta=0,645$, which corresponds to free oil concentration equal to $\mathrm{C}_{0}=\beta \cdot \mathrm{p}=$ $0,645 \cdot 867=559 \mathrm{~kg} / \mathrm{m}^{3}$.

For pressure $\mathrm{P}_{\kappa}=1,5 \mathrm{MPa}$ at the end of the screw work zone, we find the value of coefficient

$\mathrm{a}=\frac{\ln \frac{\mathrm{P}_{\mathrm{k}}}{\mathrm{P}_{\mathrm{f}}}}{\mathrm{L}_{\mathrm{f}}}=\frac{\ln \frac{1,5}{0,5}}{0,884}=1,24$

and on pressing temperature $105{ }^{\circ} \mathrm{C}$ the value of oil dynamic viscosity $\mu=0,0056 \mathrm{~Pa} \cdot \mathrm{s}$.

We use the found values and experimental value of the screw press performance $\mathrm{G}_{\mathrm{M}}=31,8 \cdot 10^{-4} \mathrm{~kg} / \mathrm{s}$.

On the basis of dependence (21) the permeability value of meal was identified $\mathrm{K}=2,9 \cdot 10^{-14} \mathrm{~m}^{2}$, the value of which does not contradict the data of this work.

Verification of the characteristics with pre-extrusion processing of raw materials followed by oil expression made it possible to simulate the filtration process. Fig. 4 shows the dependence of filtering speed change along the length of the screw channel pressing cage, obtained from formulas (1) and (3)

$$
\mathrm{V}_{\mathrm{f}}(\mathrm{z})=\frac{\mathrm{K} \cdot \mathrm{P}_{\mathrm{f}}}{\mu \cdot \mathrm{h}} \cdot \mathrm{e}^{\mathrm{az}}
$$

The results of the calculations are consistent with the data of this work. 


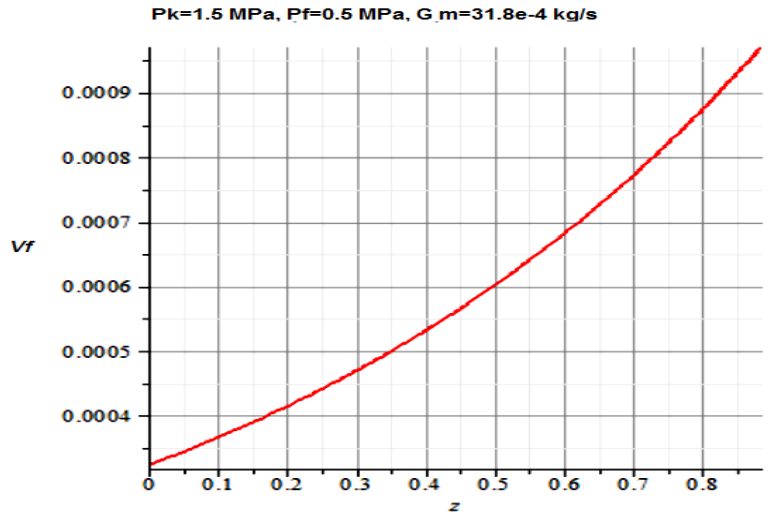

Fig. 4: Filtering Speed Change along the Length of the Screw Channel.

Fig. 5 shows the nature of the change in the free oil content in meal $\mathrm{C}_{\mathrm{Z}} / \mathrm{C}_{\mathrm{o}}$ along the length of the screw channel, obtained by equation (13).

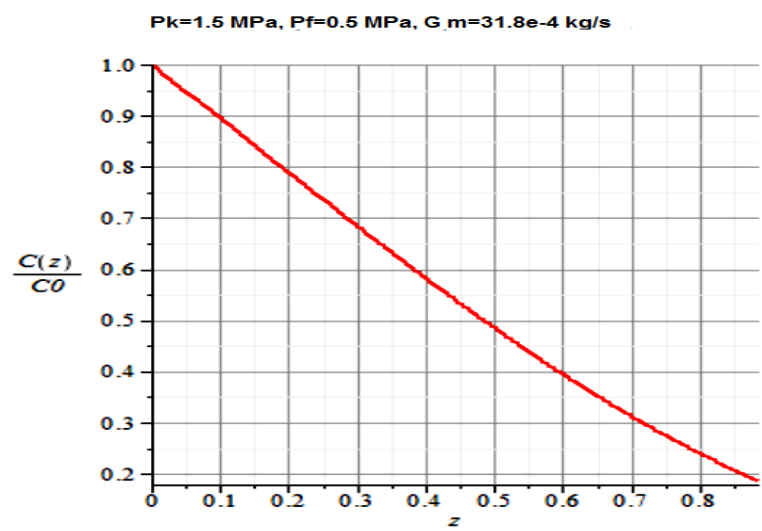

Fig. 5: Oil Content Change in Meal along the Length of the Screw Channel.

To find the change rate of the dimensionless oil concentration in the meal along the length of the pressing cage, we differentiate (13):

$\frac{\mathrm{d}\left(\frac{\mathrm{C}(\mathrm{z})}{\mathrm{Co}}\right)}{\mathrm{dz}}=-\frac{K \mathrm{P}_{\mathrm{f}} \mathrm{e}^{\mathrm{az}}}{\mu \mathrm{h}^{2} \mathrm{u}_{\mathrm{zave}}} \cdot \frac{\mathrm{e}^{\frac{-K \mathrm{Kp}_{\mathrm{f}}}{\mathrm{a} \mathrm{h}^{2} \mathrm{vzave}_{\mathrm{zaz}}}} \mathrm{e}^{\mathrm{az}}}{\mathrm{e}^{-\frac{\mathrm{Kp}}{\mathrm{a} \mathrm{h}_{\mathrm{f}}^{2}} \mathrm{v}_{\text {zave }}}}$

The results of calculations according to (27) are shown in Fig. 6. At a distance $\mathrm{z}=0,15 \mathrm{~m}$ from the beginning of the screw channel, the maximum rate of change in the oil content is observed in meal.

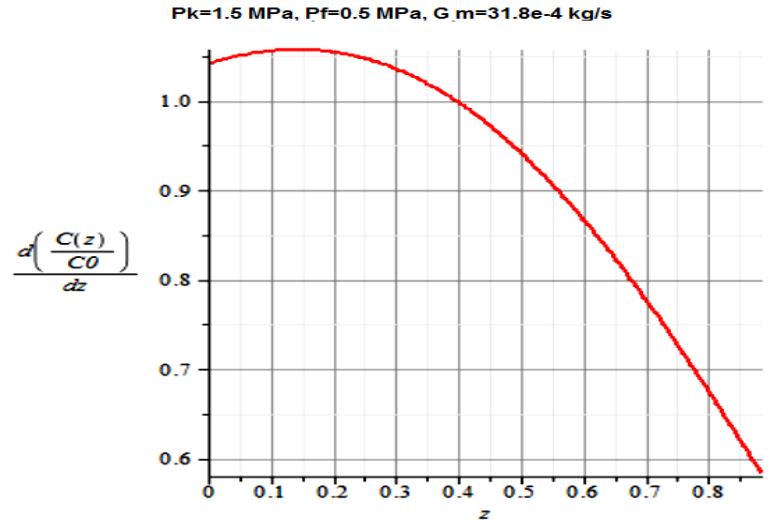

Fig. 6: Change Rate of Dimensionless Oil Concentration in Meal along the Length of the Screw Channel.

Fig. 7 shows dependence of the change in the dimensionless concentration of filtered oil along the length of the screw channel, obtained on the basis of equation (17).

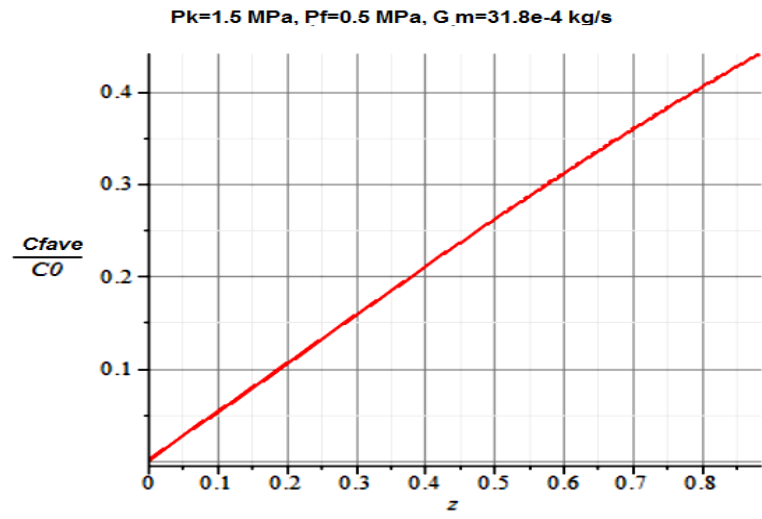

Fig. 7: Change in the Average Concentration of the Filtered Oil along the Length of the Screw Channel

Differentiating (17) on $\mathrm{z}$ we will obtain the following

$\left.\frac{\overline{C \Phi}}{C_{0}}=\frac{\left[\Gamma\left(0, \frac{K_{1}}{a}\right)-z a e^{-\frac{K_{1}}{a}}-\Gamma\left(0, \frac{K_{1}}{a} e^{a z}\right)\right] e^{\frac{K_{1}}{a}}}{z^{z_{a}}}-\frac{\left(a e^{-\frac{K_{1}}{a}}+a e^{-\frac{K_{1}}{a} c^{a z}}\right)}{z a}\right] e^{\frac{K_{1}}{a}}$,

Where $K_{1}=\frac{\mathrm{KP}_{\mathrm{f}}}{\mu \mathrm{h}^{2} \omega_{\mathrm{zcp}}}$

The results of the calculations in accordance with (28) are obtained in Fig.8. Note that in this case the maximum value of the function is shifted towards higher values of $\mathrm{z}\left(\mathrm{z}_{\max } \approx 0,2\right)$ comparing to fig. 6

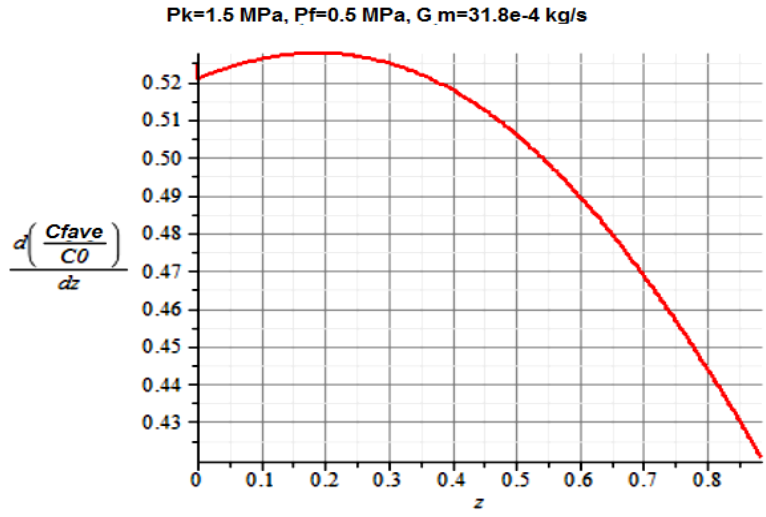

Fig. 8: Change Rate of the Dimensionless Average Concentration of the Filtered Oil along the Length of the Screw Channel.

Note that in the example of calculation, fraction of the unfiltered oil at the outlet from expression zone is $\mathrm{C}\left(\mathrm{L}_{\mathrm{f}}\right) / \mathrm{C}_{\mathrm{o}}=0,187$.

\section{Conclusion}

Thus, the developed mathematical model of oil expression in a screw press meets physical meaning of the phenomena and can be used to analyze the presses operations, as well as their design. The obtained solutions were verified by the machine method for adequacy to the actual pressing process. The error of the calculated data from the experimental values according to the absolute value did not exceed $15 \%$.

As a result, it was found that the productivity of a screw oil press depends on the assembly of design parameters of the screw shaft, pressing cage, and on physico-mechanical properties of the pressed material. It is revealed that the main amount of oil is expressed at the first and second stages of the pressing cage. The meal skeleton has the ability to resist deformation and reduce the porosity, resulting in the reduced oil output.

Thus, analysis of mathematical model and realization of constructive solutions that it provides will allow:

- To exclude the possibility of heating the chamber with electric heaters due to the effect of dissipative forces; 
- To obtain a wide range of vegetable oils from oil raw materials due to the adjustable size of the gap between the plates;

- $\quad$ To reduce metal intensity, due to singularity of the pressing cage design; - To ensure alignment of the pressing plates connection with each other and guarantee reliability of the assembly as a consequence; - to intensify the process of pressing oil out of oil seeds through the selection of the optimum parameters of the working chamber and adjustable gap for the outflow of the oil cake. - To adjust the oil press settings for a wide range of oil crops due to the possibility of adjusting the gap between the plates,

- To reduce the amount of pulp in the expressed oil.

The results of scientific research will influence the development of scientific, technical and technological areas in the field of creating new food products with programmable properties (including therapeutic and preventive action). The developmental work based on those results will reveal new technological solutions in the field of creation of the new technological equipment for various branches of the food industry. The developed recommendations and proposals for the use of the results have already been applied in the ongoing innovative projects for the construction of oil presses and other equipment.

\section{References}

[1] V.N. Vasilenko Investigation of the kinetic regularities of the extraction of vegetable oils in a screw oilpress [Text] / V. N. Vasilenko M.V. Kopylov // Bulletin of Voronezh State University of Engineering Technologies 2012. № 1. P. 10-12.

[2] Development of theoretical and technological bases for complex processing of oilseeds: Monograph/ V. N. Vasilenko, L. N. Frolova, I V. Dragan. Voronezh: VSUET, 2014. p. 148.

[3] L.N. Frolova Modeling of the process of oscillating drying of oil crops on the example of camelina oil seeds [Text] / L. N. Frolova, I. V. Dragan, Yu.A.Tarkaev, S.A. Shevtsov / / Storage and processing of agricultural raw materials. - 2014. - № 4. - p. 31-34.

[4] Sokolovskyy J. Mathematical modeling of the two-dimentiona moisture and viscoelasticity states of wood in the process of drying/ J. Sokolovskyy , M. Dendiuk, A. Bakalets // Forest as renewable source of vital values for changing world: IAWS plenary meeting and conference. - S.-Petersburg-Moscow, 2009. - P. 34.

[5] Gurta Es Seyhan, F. Low temperature mushroom (A. bisporus) drying with desiccant dehumidifiers [Text] / F. Gurta Es Seyhan, O. Evranuz // Drying Technology. - 2000. - № 18. -P. 433 - 445.

[6] R.M. Davies Engineering Properties of Three Varieties of Melon Seeds as Potentials for Development of Melon Processing Machines/ Advance Journal of Food Science and Technology 2(1):63-66, 2010: ISSN: 2042-4876.

[7] S.K. Shittu, V.I.O. Ndrika. Development and performance tests of a melon (egusi) seed shelling machine. Agric Eng Int: CIGR Journal, 14(1): Manuscript 2027.

[8] http://www.waset.org/publications/10002551/comparative-studyof-the-effects-of-process-parameters-on-the-yield-of-oil-frommelon-seed-cococynthis-citrullus-and-coconut-fruit-cocos-nucifera-

[9] An Addo and A. Bart-Plange Kinetics of water sorption by egus melon (Cucumeropsis edulis) seeds / ARPN Journal of Agricultural and Biological Science. VOL. 4, NO. 6, NOVEMBER 2009: ISSN 1990-6145.

[10] V. N. Vasilenko M. V. Kopilov A.V. Nakraynikova Creation of a CAD system, "Maslopress"//Bulletin of Engineering 2012. № 2. C. 35-36.

[11] Processes and devices of food production text book for universities [Text] / A.N. Ostrikov, O.V. Abramov, A.V. Loginov, Yu.V. Krasovitsky, V.N. Vasilenko, -SPb. GIORD, 2012. - 616 p. 